\title{
Hematological Characteristics of Rats Spontaneously Developing Eosinophilia
}

\author{
Kiyoshi MATSUMOTO, Norifumi MATSUSHITA, \\ Hiroshi TOMOZAWA, and Yoh-ichi TAGAWA
}

\author{
Institute of Experimental Animals, Shinshu University School of Medicine, \\ 3-1-1 Asahi, Matsumoto, Nagano 390-8621, Japan
}

\begin{abstract}
Hematological and genetic characteristics of newly found eosinophilic rats were studied. Hematologically, high blood eosinophil counts started at 6 weeks of age. Almost all

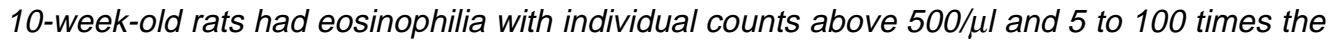
normal level. Proliferating eosinophils had normal morphology. An increase in lymphocyte counts was observed at 5 weeks of age, one week earlier than the onset of eosinophilosis. In bone marrow, proliferation of eosinophils was also observed at 8 weeks of age and thereafter progressed, suggesting a role in the pathogenesis of eosinophilia in this rat. The results of genetic cross experiments revealed the disease to be hereditary. The spontaneously eosinophilic rat therefore warrants attention as a model for studying the underlying mechanisms of human and animal eosinophilia.
\end{abstract}

Key words: eosinophilia, hematology, model animal, rat

\section{Introduction}

Eosinophils are leukocytes derived from bone marrow. In humans, eosinophilia is commonly seen in allergic, parasitic, infectious, pulmonary, dermatologic, neoplastic, and immunodeficiency diseases. In almost all cases, the pathophysiology is the same: tissue deposition of eosinophils and release of eosinophilic granule products that cause tissue and organ damage [5].

Several animal models for eosinophilia have been studied; for example, mice infected with parasites [10], or bone marrow eosinophilia due to Aspergillus infection [8], but, to our knowledge, no mutant rats with eosinophilia have hitherto been reported.
At the end of 1997, among SPF SD (Sprague-Dawley) rats purchased from commercial breeder (Japan SLC Inc., Shizuoka, Japan) we found a pregnant one with eosinophilia. Three males and five females from the pregnant rat had high eosinophil counts (more than 360 cells $/ \mu l$ ) at 10 weeks of age. Thereafter, the animals were maintained by brother-sister matings with the criteria (more than 850 eosinophils $/ \mu l$ at 10 weeks of age) for the selection of animals. This rat with eosinophilia was named Matsumoto Eosinophilia Shinshu (MES) [7] and they are now in the 7th generation. The present study was conducted to investigate their hematological characteristics. In addition bone marrow cells were examined and age and genetic cross experiments were performed.

(Received 29 October 1999 / Accepted 5 April 2000)

Address corresponding: K. Matsumoto, Institute of Experimental Animals, Shinshu University School of Medicine, 3-1-1 Asahi, Matsumoto, Nagano 390-8621, Japan 


\section{Materials and Methods}

Animals: Male and female MES rats in F3 and F4 generations were used for the present study. For genetic cross experiments, Slc:SD and ACI/N Slc rats of both sexes were purchased from Japan SLC Inc. All the rats were kept in our facilities and were free of the following microorganisms: Pseudomonas aerginosa, Pasteurella pneumotropica, Bordetella bronchiseptica, Salmonella Spp., Corynebacterium kutscheri, Tyzzer's organism (Clostridium piliformis), Dermatophytes, $M y$ coplasma pulmonis, hemagglutinating virus of Japan, the mouse hepatitis virus, Giardia spp., Spironucleus muris and Syphacia. Aspergillus infection which causes eosinophilia was not detected in the MES rats as determined by histopathological examination.

The animal room was controlled at $24 \pm 2^{\circ} \mathrm{C}$ and 55 $\pm 10 \%$ relative humidity, with a complete ventilatory exchange of fresh air 15 times/hr and a $12 \mathrm{hr}$ lightdark-cycle. The rats were housed in polycarbonate cages and fed a commercial diet (MF; Oriental Yeast Co. Ltd., Tokyo, Japan) and water ad libitum.

This study was carried out in accordance with the Guidelines for Animal Experimentation of the Shinshu University School of Medicine.

Hematological examination: Animals were held in the hand without anesthesia and blood samples were collected from the jugular vein for hematology. The number of leukocytes was counted in an automated cell counter (Sysmex F-820, Toa Medical Electronics Co. Ltd., Kobe, Japan), and differential leukocyte counts (\%) were performed on Wright stained blood films. The absolute numbers of lymphocytes and eosinophils (cells $/ \mu l$ ) were calculated. The animals were anesthetized with ether and killed by cervical dislocation and femoral bone marrow cells and differential cell counts were estimated by the method reported previously [6].

Male ACI rats were crossed with female MES rats to obtain F1 (MES $\times$ ACI) rats. An F2 intercross was then performed by mating F1 hybrid siblings, followed by a backcross with female MES rats $(\mathrm{MES} \times \mathrm{F} 1)$. The numbers of blood eosinophils in offspring obtained by cross experiments were counted at 10 weeks of age.

\section{Results}

Regarding their general condition, MES rats were healthy and had normal reproductive performance through the 7 generations, although wryneck was sometimes observed. So far one male and two females have died of anemia between 18 and 25 weeks of age. The body weight of MES rats of both sexes was found to be about 20\% lower than that of normal SD rats (Fig. 1).

Absolute eosinophil and lymphocyte counts in the peripheral blood of MES rats at different ages are shown in Figs. 2 and 3. An increase in the number of lymphocytes was noted at 5 weeks of age and of eosinophils at 6 weeks, with continued increase thereafter. Eosinophil counts of MES rats at 10 weeks of age were 5- to 100-times higher than those of normal SD rats and were higher in females than in males. Blood smears of MES rats demonstrated normal mature eosinophils with typical morphology.

In bone marrow, proliferation of eosinophils was observed at 8 weeks of age and increased thereafter (Fig. 4). An increase in the $M / E$ ratio (myeloids/erythroids) due to a reduction in the number of erythroblasts and an increase in eosinophils with age were also found. No obvious changes were apparent in counts of lymphocytes or other cells. In hematology and bone marrow cells, no clear differences between generations were observed (data not shown).

To detect the genetic background of MES rats, a genetic cross experiment was performed. The number of eosinophils in 10-week-old rats was counted for 31 SD (normal), 21 ACI (normal), 27 MES, 19 F1 intercrosses $($ MES $\times$ normal ACI), 23 F2 $(\mathrm{F} 1 \times$ F1 $)$ and 29 backcrosses $(\mathrm{F} 1 \times \mathrm{MES})$, and the results are shown in Fig. 5. All SD, ACI, and F1 rats had eosinophil counts less than $120 / \mu l$ (normal level). All the MES rats had counts between 516 and 7600/ $\mu l$ except one which also had eosinophilia $(3680 / \mu l)$ at 16 weeks of age. Among the F2 $(\mathrm{F} 1 \times \mathrm{F} 1)$ animals, $78 \%$ had eosinophil counts less than $120 / \mu l$, but the others (22\%) had slightly higher values, between 122 and 460/ $\mu$ l. In F2 backcrosses (F1 $\times$ MES), $48 \%$ had eosinophil counts less than $120 / \mu$ l, $24 \%$ had between 138 and $360 / \mu l$, and $28 \%$ of the rats had between 594 and 5292/ $\mu l$, respectively.

\section{Discussion}

The mean normal value for blood eosinophils is 200/ $\mu l$ in humans and $80 / \mu l$ in rats (our control data for 120 SD rats of both sexes). A patient who has an absolute 
(g)

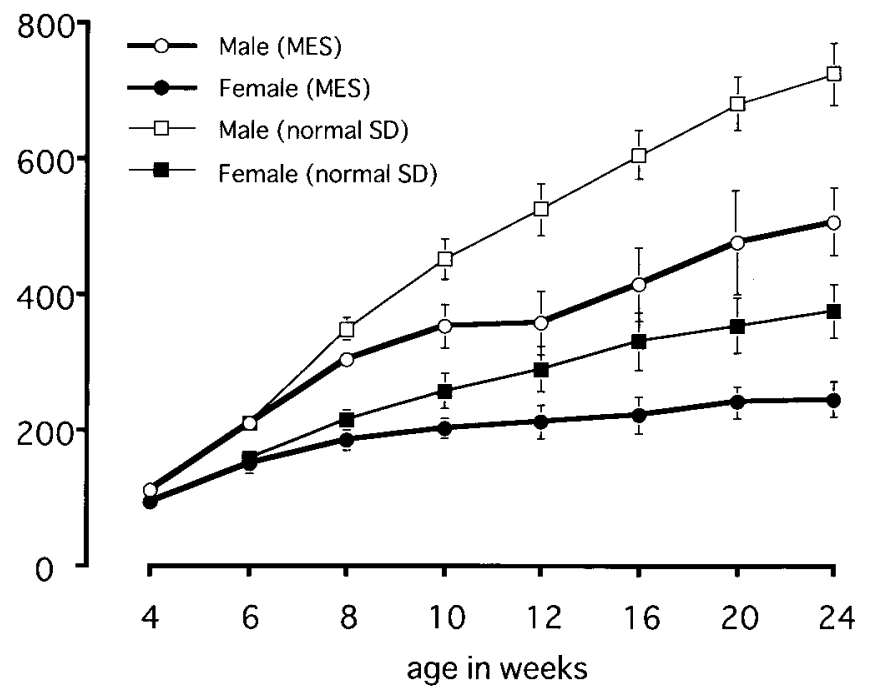

Fig. 1. Body weight curves for MES and normal SD rats.

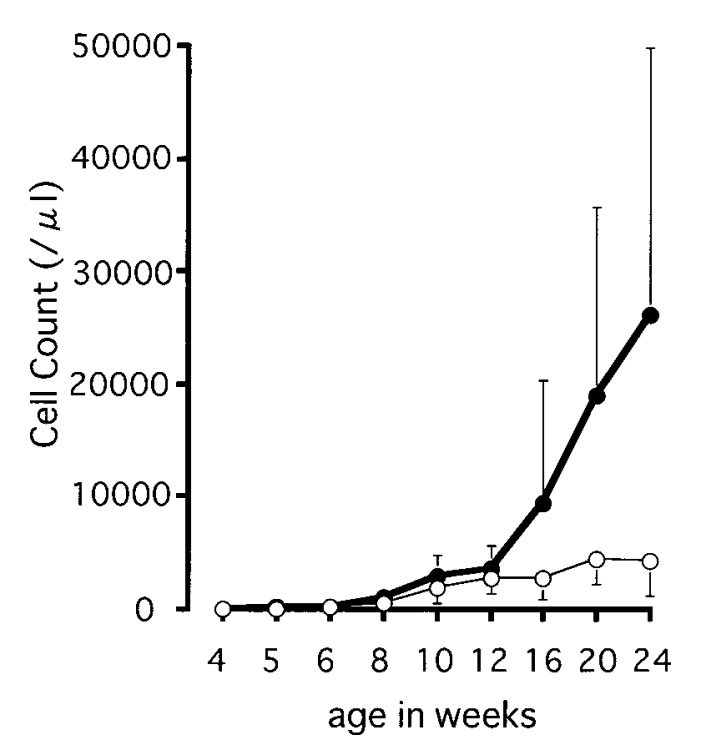

Fig. 2. Age-associated changes in numbers of eosinophils in blood from MES rats of both sexes. Each bar ( $\bigcirc$ : male, female) represents the mean \pm SD for 27 animals of the F3 and F4 generations. Normal blood eosinophil counts of SD rats are in the range of 30 to $120 / \mu l$.

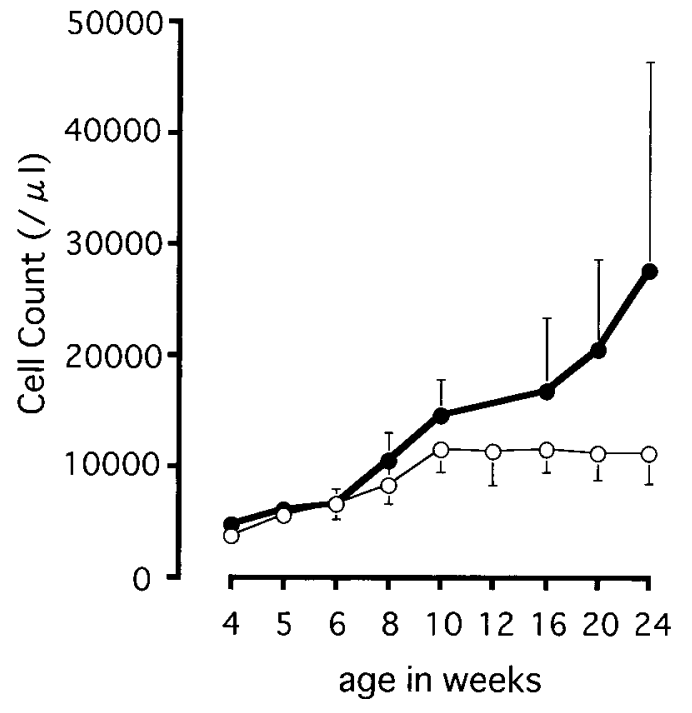

Fig. 3. Age-associated changes in numbers of lymphocytes in blood from MES rats of both sexes. Each bar ( $\mathrm{O}$ : male, female) represents the mean \pm SD for 27 animals of the F3 and F4 generations. Normal blood lymphocyte counts of SD rats are in the range of 4000 to $6000 / \mu l$. blood eosinophil count of more than $450-500 / \mu l$ is generally diagnosed as suffering from eosinophilia [4].

In the present study, eosinophil counts in $83 \%$ of male and $98 \%$ of female MES rats at 10 weeks of age were more than 500 eosinophils/ $\mu l$. Additional hema- tological tests revealed that eosinophil levels in several rats less than $500 / \mu l$ at 10 weeks, subsequently increased and were within the eosinophilic range at 16-week of age (data not shown). The mean eosinophil counts of MES rats were generally higher in females than in 


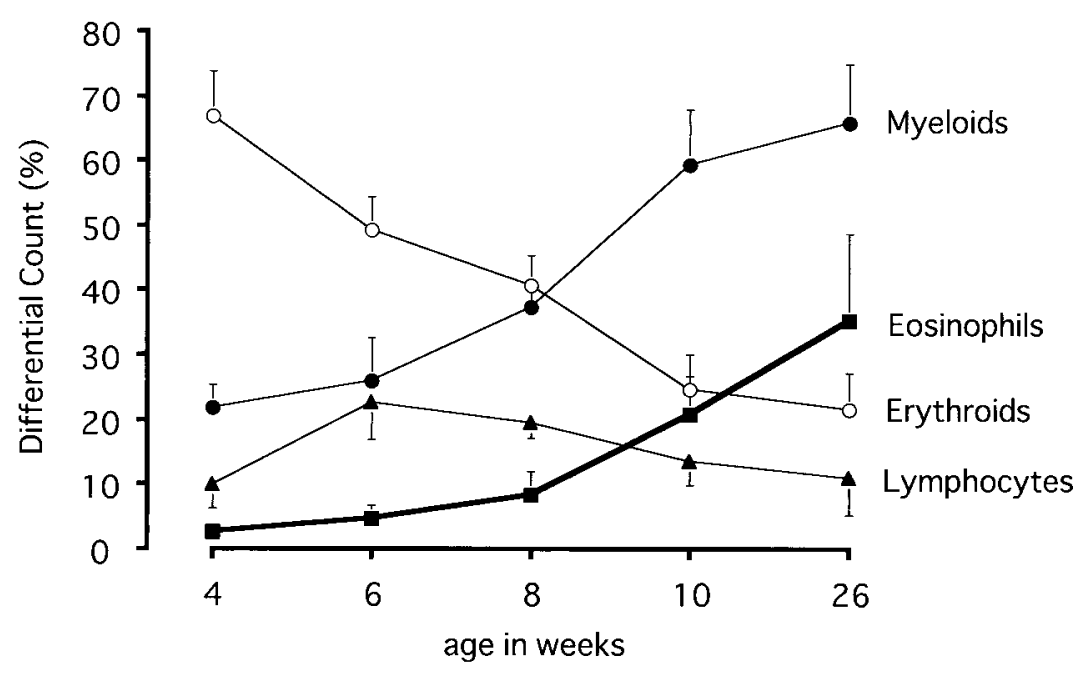

Fig. 4. Age-associated changes in the differential cell counts in bone marrow of MES rats. Each bar represents the mean \pm SD for 5 female rats dissected at 4, 6, 8, 10 and 26 weeks of age. The normal value for bone marrow eosinophils in SD rats is about $3 \%$.

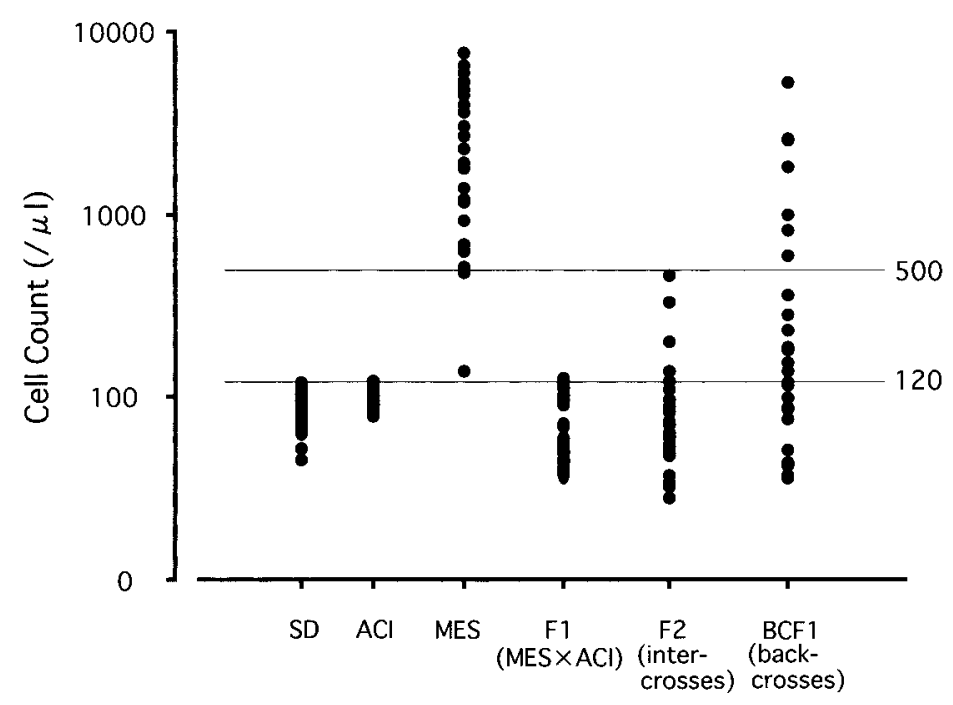

Fig. 5. The absolute numbers of eosinophils in blood from SD (normal), ACI (normal), MES, F1 (MES $\times$ ACI) and F2 (intercrosses and backcrosses) rats of both sexes at 10 weeks of age. The higher limit of the eosinophil count in normal rats is $120 / \mu l$ and the lower limit for the diagnosis of eosinophilia is $500 / \mu l$.

males, but eosinophil levels in both sexes were much higher than in normal SD rats. In females, wide distribution of the eosinophil count was observed after 16 weeks of age. The reasons for the scatter are still unclear, although the degree of lymphocyte proliferation in lymph nodes may relate to the eosinophil counts. Therefore, all of the MES rats could be diagnosed as having eosinophilia. The morphology of peripheral blood eosinophils in MES rats was normal, and no immature cells and/or cells with abnormal morphology 
suggestive of eosinophilic leukemia were encountered. Eosinophilic leukemia is actually considered to be a very rare and poorly defined entity in humans, and there have been no reports of this condition in rats.

Eosinophilia is one complication of lymphoproliferative disorders in man [1], and in this study blood lymphocytes were increased one week earlier than the onset of eosinophilosis, suggesting that their proliferation is related to the pathogenesis of eosinophilia [11] in MES rats.

Histopathologically, infiltrations of eosinophils into the stomach, intestines, spleen, lungs and lymph nodes were observed in all MES rats over 12 weeks of age. And granuloma of mesenteric lymph nodes, eosinophilic gastritis and aortitis, where eosinophilic materials were detected, were frequently found ([7], data shown elsewhere).

Our animals were maintained under SPF (specificpathogen free) conditions and were also free from Aspergillus fumigatus infection which is known to cause eosinophilia. Thus the cause of eosinophilia is neither infection nor leukemia. The significance of the occasional appearance of wryneck is still unclear.

In bone marrow obvious proliferation of eosinophils was found, and is known to be regulated by cytokines such as IL-3, IL-5 and CSF-GM [2, 4]. It has also been reported that IL-5 is involved in the pathogenesis of hyper eosinophilic syndrome (HES) and secondary eosinophilia [2, 9].

In a genetic cross experiment, eosinophilia $(>500 / \mu l)$ was observed in almost all MES rats and $28 \%$ of F2 backcrosses. A slight increase in the eosinophil count, $120-500 / \mu l$, was noted in $22 \%$ of $\mathrm{F} 2$ intercrosses and $24 \%$ of F2 backcrosses, whereas all of the F1 rats were in the normal range, $\langle 120 / \mu l$, suggests that heritable factors may be related to the pathogenesis of eosinophilia in the MES rat. In humans, however, there have been no reports of hereditary eosinophilia or a hypereosinophilic syndrome [3].

In conclusion the presently described hematological characteristics of the MES rat which develops spontaneous eosinophilia point to its potential as a model animal for research on the regulatory mechanisms of eosinophilia and for human eosinophilia.

\section{References}

1. Bastern, A. and Beeson, P.B. 1970. Mechanism of eosinophilia. II. Role of the lymphocyte. J. Exp. Med. 131: 1288-1305.

2. Enokihara, H., Kajitani, H., Nagashima, S., Tsunogake, S., Takano, N., Saito, K., Furusawa, S., Shishido, H., and Takatsu, K. 1990. Interleukin 5 activity in sera from patients with eosinophilia. Br. J. Haematol. 75: 458-462.

3. Fauci, A.S., Harley, J.B., Roberts, W.C., Ferrans, V.J., Gralnick, H.R., and Bjornson, B.H. 1982. NIH conference. The idiopathic hypereosinophilic syndrome. Clinical, pathophysiologic, and therapeutic considerations. Ann. Intern. Med. 97: 78-92.

4. Franklin, D.Z. 1993. Eosinopenia and eosinophilia. pp. 845849. In: Hematology 4th Ed. (Williams, W. J., Beutler, E., Erslev, A.J., and Lichtman, M. A.eds.), Mc-Graw-Hill Pub. Co., New York.

5. Gleich, G. and Adolphson, C. 1986. The eosinophilic leukocyte: structure and function. Adv. Immunol. 39: 177188.

6. Matsumoto, K., Inukai, S., Isaka, T., Aruga, N., Nakamura, S., Nagasawa, T., and Naito, J. 1995. Cell counts in peripheral blood and bone marrow of male C.B.17 scid/ scid mice. Lab. Anim. 29: 218-222.

7. Matsumoto, K., Matsushita, F., and Umemura, T. 1999. A new rat model of eosinophilia. Rat Genome 5: 11-14.

8. Murali, P.S., Kurup, V.P., Guo, J., and Fink, J.N. 1997. Development of bone marrow eosinophilia in mice induced by aspergillus fumigatus antigens. Clin. Immunol. Immunopathol. 84: 216-220.

9. Owen, W.F., Rothenberg, M.E., Petersen, J., Weller, P.F., Silberstein, D., Sheffer, A.L., Soberman, R.J., and Austen, K.F. 1989. Interleukin 5 and phenotypically altered eosinophils in the blood of patients with idiopathic hypereosinophilic syndrome. J. Exp. Med. 170: 343-348.

10. Takamoto, M., Wang, Z.X., Watanabe, N., Matsuzawa, A., Nariuchi, H., and Sugane, K. 1998. Eosinophilia, IgE production, and cytokine production by lung $\mathrm{T}$ cells in surface CD4-deficient mutant mice infected with Toxocaracanis. Immunology 95: 97-104.

11. Thomson, A.W., Mathie, I.H., and Sewell, H.F. 1987. Cyclophosphamide-induced eosinophilia in the rat: Concominant changes in T-cell subsets, B cells and large granular lymphocytes within lymphoid tissues. Immunology 60: 383-388. 